\title{
Ensino das Práticas Integrativas e Complementares nos cursos de enfermagem do Estado de São Paulo
}

\begin{abstract}
RESUMO | Objetivo: analisar a oferta de disciplinas sobre as Práticas Integrativas e Complementares nos cursos de graduação em Enfermagem no Estado de São Paulo. Método: estudo descrito e retrospectivo com abordagem quantitativa. A coleta de dados foi realizada por meio da busca nas páginas web das instituições afim de coletar os dados das grades curriculares. Foi realizada a análise descritiva dos dados. Resultados: foram selecionadas 172 instituições de ensino superior no Estado de São Paulo sendo 102 (59,3 \%) localizadas no interior do Estado, 152 (88,4\%) instituições privadas de ensino, 31 (18\%) apresentaram na grade curricular disciplinas relacionadas a Práticas Integrativas e Complementares, destas disciplinas 17 (9,9\%) eram obrigatórias, 66 (38,4\%) presenciais e com carga horária média de 403,6 horas ( $\mathrm{DP}=411,8$ ). Conclusões: ainda há uma escassez de instituições públicas e privadas do Estado de São Paulo que oferecem disciplinas nos cursos de graduação em Enfermagem sobre Práticas Integrativas e Complementares.
\end{abstract}

Palavras-chaves: Terapias Complementares; Práticas Integrativas e Complementares; Ensino; Enfermagem.

\begin{abstract}
Objective: to analyze the offer of disciplines on Integrative and Complementary Practices in undergraduate nursing courses in the State of São Paulo. Method: described and retrospective study with a quantitative approach. Data collection was carried out by searching the institutions' web pages to collect data from the curriculum. Descriptive data analysis was performed. Results: 172 higher education institutions were selected in the State of São Paulo, 102 (59.3\%) located in the interior of the State, $152(88.4 \%)$ private educational institutions, $31(18 \%)$ presented subjects in the curriculum related to Integrative and Complementary Practices, of these disciplines 17 (9.9\%) were mandatory, 66 (38.4\%) in person and with an average workload of 403.6 hours (SD $=411.8$ ). Conclusions: there is still a shortage of public and private institutions in the State of São Paulo that offer disciplines in undergraduate nursing courses on Integrative and Complementary Practices.
\end{abstract}

Keywords: Complementary Therapies; Integrative and Complementary Practices; Complementary Medicine; Teaching; Nursing.

RESUMEN | Objetivo: analizar la oferta de disciplinas Prácticas Integradoras y Complementarias en cursos de grado en enfermería en el Estado de São Paulo. Método: estudio descrito y retrospectivo con enfoque cuantitativo. La recogida de los datos se realizó mediante la búsqueda en las páginas web de las instituciones para recopilar datos del plan de estudios. Se realizó un análisis descriptivo de los datos. Resultados: 172 instituciones de educación superior fueron seleccionadas en el Estado de São Paulo, 102 (59.3\%) ubicadas en el interior del Estado, 152 (88.4\%) instituciones educativas privadas, 31 (18\%) presentaron materias en el plan de estudios relacionadas con las Prácticas Integradoras y Complementarias, de estas disciplinas 17 (9.9\%) eran obligatorias, 66 (38.4\%) en persona y con una carga de trabajo promedio de 403.6 horas $(D E=411.8)$. Conclusiones: todavía hay una escasez de instituciones públicas y privadas en el Estado de São Paulo que ofrecen disciplinas en cursos de grado en enfermería sobre Prácticas Integradoras y Complementarias.

Palavras claves: Terapias Complementarias; Prácticas Integradoras y Complementarias; Enseñanza; Enfermería.

\section{Tatiane Alves Oliveira}

Bacharel em Enfermagem pela Universidade Guarulhos (UNG). https://orcid.org/00000002-0096-2651

\section{Tereza Alinne Araújo de Assis}

Bacharel em Enfermagem pela Universidade Guarulhos (UNG). https://orcid.org/00000002-9799-4253

\section{Jefferson Costa Macedo}

Bacharel em Enfermagem pela Universidade Guarulhos (UNG). https://orcid.org/00000001-8184-4288

\section{Ingryd de Araújo Silva}

Bacharel em Enfermagem pela Universidade Guarulhos (UNG). https://orcid.org/00000002-7838-2884

\section{Elaine Avelar de Almeida}

Enfermeira. Docente do Senviço Nacional de Aprendizagem Comercial (SENAC). Discente de Mestrado do Programa de Pós-graduação em Enfermagem da Universidade Guarulhos (UNG). https://orcid.org/0000-0002-0242-5198

\section{Noélle de Oliveira Freitas}

Enfermeira. Docente do Programa de PósGraduação em Enfermagem da Universidade Guarulhos (UNG). https://orcid.org/00000001-6742-5247

Recebido em: 10/06/2020
Aprovado em: 11/06/2020

INTRODUÇÃO

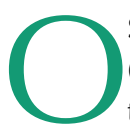
Sistema Único de Saúde (SUS) com a Lei n. ${ }^{\circ} 8.080 / 90$ transformou a assistência em saúde no Brasil com seus princípios da universalidade, equidade e integralidade, e com a ideia de descentralizar a política administrativa, além de inserir a sociedade como parte integrante deste sistema ${ }^{(1)}$.

Com proposta do SUS de uma assistência com foco na prevenção, promoção e não somente na cura da doença, surgiram novas perspectivas para o cuidado. A Política Nacional das Práticas Integrativas e Complementares (PNPIC), em 2006 por meio da Portaria GM/MS n. ${ }^{\circ}$ 971, aprovou as práticas na assistência tendo como foco principal a prevenção de agravos nas doenças crônicas, promoção, proteção e recuperação da saúde, baseada no modelo de atenção humanizada e centrada na integralidade do indivíduo. A PNPIC, proposta em 2006, iniciou o programa com cinco práticas terapêuticas: homeopatia, medicina tradicional chinesa/ acupuntura, plantas medicinais e fitoterapia e o observatório de medicina antroposófica e termalismo social/ crenoterapia $^{(2)}$.

A Portaria n. ${ }^{\circ} 849$ de 2017 acrescentou a esta política 14 novas Práticas Integrativas e Complementares (PICs): arteterapia, ayurveda, biodança, dança 
circular, meditação, musicoterapia, naturopatia, osteopatia, quiropraxia, reflexoterapia, reiki, shantala, terapia comunitária integrativa e yoga ${ }^{(3)}$. Em março de 2018, foram implantadas mais 14 PICs por meio da Portaria n. ${ }^{\circ} 702$, sendo: aromoterapia, apiterapia, bioenergética, constelação familiar, cromoterapia, geoterapia, hipnoterapia, imposição de mãos, medicina antroposófica/ antroposifia aplicada a saúde, ozonioterapia, terapia de florais e termalismo social/ crenoterapia $^{(4)}$. Sendo assim, atualmente o SUS, por meio do PNPIC, regulamenta 33 tipos de PICs com o objetivo de garantir uma assistência humanizada, preventiva e com ênfase na recuperação da saúde.

Com a PNPIC, a população pode ter acesso a diversas PICs, fortalecendo um dos princípios dos SUS, pois atende aos usuários individualmente em uma assistência humanizada, sendo que este usuário torna-se corresponsável por seu processo de saúde-doença ${ }^{(2)}$.

O Conselho Federal de Enfermagem (COFEN) por meio da Resolução n. ${ }^{\circ} 0500$ de 2015 reconheceu as terapias alternativas como especialidade da Enfermagem garantido ao enfermeiro o direito de atuar desde que esteja apto e qualificado ${ }^{(5)}$. Em 11 de julho de 2018, o COFEN, por meio da Resolução n. ${ }^{\circ}$ 581/2018, considera obrigatório o registro dos títulos de pós-graduação Latu Sensu e Stricto Sensu dos enfermeiros em seus conselhos regionais de sua jurisdição. Sendo assim, as PICs tornam-se uma especialidade da Enfermagem $^{(6)}$. Embora as PICs tenham sido consideradas como especialidade da enfermagem, ainda há uma deficiência do ensino sobre esta temática nos cursos de graduação.

Um estudo avaliou as disciplinas sobre PICs oferecidas nos cursos de Enfermagem de instituições públicas de ensino do Brasil e identificou que entre as 87 instituições, 23 (26,1\%) ofereciam disciplinas relacionadas às PICs, sendo a maioria de caráter optativo, totalizando $17-73,9 \%{ }^{(7)}$. Este estudo revelou que existem muitas lacunas na oferta dos cur- sos, e ainda há muitos profissionais que finalizam a formação acadêmica sem terem contato com o tema sobre $\mathrm{PICs}^{(7)}$.

Em 2018, um estudo avaliou os cursos de graduação e de pós-graduação de instituições públicas no Estado do Rio de Janeiro e identificou que entre as 15 áreas da saúde pesquisadas foram encontradas 46 opções de disciplinas, sendo 39 disciplinas da graduação e sete estavam vinculadas à pós-graduação no Estado do Rio de Janeiro ${ }^{(8)}$. O curso de Medicina se destaca apresentando o maior número de opções chegando a $31 \%$, já a Enfermagem ficou em terceiro lugar com $14 \%$, sendo que deste percentual, 17 disciplinas são obrigatórias 19 optativas e 10 são eletivas ${ }^{(8)}$.

Este estudo teve como foco os cursos de graduação em Enfermagem das instituições de ensino superior públicas e privadas do Estado de São Paulo a fim de identificar o quadro atual da oferta de de disciplinas com o foco nas PICs. Assim, este estudo teve como objetivo avaliar a oferta de disciplinas sobre as PICs nos cursos de graduação e pós-graduação lato sensu em Enfermagem no Estado de São Paulo.

\section{METODOLOGIA}

Trata-se de um estudo descritivo e retrospectivo com abordagem quantitativa.

A lista de instituições do Estado de São Paulo foi extraída da página web do Ministério da Educação - $\mathrm{MEC}^{(9)}$. A coleta de dados foi realizada nos meses de julho a agosto de 2019 por meio das páginas web das instituições públicas e privadas do Estado de São Paulo a fim de identificar a grade curricular dos cursos de graduação em Enfermagem. Além disso, foi verificado se a instituição oferecia curso de pós-graduação lato sensu e a grade curricular destes cursos.

Para coleta de dados foi utilizado um formulário com os seguintes dados: nome da instituição, tipo de instituição (pública ou privada), nome da disciplina, data de criação da disciplina, tipo 
Tabela 1. Distribuição das características das universidades selecionadas no estudo ( $n=172$ ). Guarulhos, SP, Brasil, 2019.

\begin{tabular}{lcc|} 
Variáveis & N & $(\%)$ \\
\hline Região & & \\
\hline Capital & 47 & $(27,3)$ \\
\hline Região Metropolitana & 23 & $(13,4)$ \\
\hline Interior & 102 & $(59,3)$ \\
\hline Tipo de curso & & \\
\hline Bacharelado & 151 & $(87,8)$ \\
\hline Licenciatura & 1 & $(0,6)$ \\
\hline Bacharelado e Licenciatura & 1 & $(0,6)$ \\
\hline Pós - Graduação & 17 & $(9,9)$ \\
\hline Sem informação & 2 & $(1,2)$ \\
\hline Tipo Instituição & & \\
\hline Privada & 152 & $(88,4)$ \\
\hline Pública Estadual & 10 & $(5,8)$ \\
\hline Pública Municipal & 6 & $(3,5)$ \\
\hline Pública Federal & 2 & $(1,2)$ \\
\hline
\end{tabular}

Tabela 2. Distribuição das características das disciplinas sobre práticas integrativas e complementares $(n=172)$. Guarulhos, SP, Brasil, 2019.

\begin{tabular}{|c|c|c|c|c|c|c|}
\hline Variáveis & N & $(\%)$ & Mediana & (Intervalo) & Média & $(D P)^{*}$ \\
\hline \multicolumn{7}{|l|}{ Oferecem a disciplina } \\
\hline Não & 139 & $(80,8)$ & & & & \\
\hline Sim & 31 & $(18,0)$ & & & & \\
\hline Sem informação & 2 & $(1,2)$ & & & & \\
\hline \multicolumn{7}{|l|}{ Tipo de Disciplina } \\
\hline Obrigatória & 17 & $(9,9)$ & 11 & & 15 & 5,7 \\
\hline Optativa & 15 & $(8,7)$ & & & & \\
\hline Sem informação & 140 & $(81,4)$ & & & & \\
\hline \multicolumn{7}{|l|}{ Modalidade } \\
\hline Presencial & 66 & $(38,4)$ & 1 & & 35,5 & 48,8 \\
\hline EADt & 1 & $(0,6)$ & & & & \\
\hline Sem informação & 105 & $(61,0)$ & & & & \\
\hline Carga Horária (em horas) & 21 & & 360 & $(36-1200)$ & 403,6 & 411,8 \\
\hline
\end{tabular}

Nota: *Desvio-padrão; †Ensino a distância

de disciplina (obrigatória ou optativa) e carga horária.

De acordo com a Resolução n. ${ }^{\circ} 510$ de 2016 do Conselho Nacional de Saúde - CNS(10), o estudo dispensa avaliação de um Comitê de Ética em Pesquisa de Seres Humanos, pois utiliza informações de domínio público.

Os dados coletados foram digitados no programa Microsoft Excel ${ }^{\circledR}$ versão
2016 e analisados no programa Statistical Package for the Social Sciences $®$ (SPSS) versão 20 para Windows ${ }^{\circledR}$. Foi realizada a análise descritiva dos dados por meio de frequências absolutas e relativas, medidas de tendência central (média) e dispersão (desvio-padrão).

\section{RESULTADOS}

Foram identificadas 176 instituições de ensino no Estado de São Paulo, entre as quais quatro não disponibilizavam as informações na página web.

Sendo assim, totalizaram 172 instituições que ofereciam o curso de Enfermagem no Estado de São Paulo. Entre as instituições encontradas 102 (59,3 $\%)$ estavam localizadas no interior do Estado, 151 (87,8\%) ofereciam o curso de bacharelado em Enfermagem e 152 $(88,4 \%)$ eram instituições privadas de ensino (Tabela 1).

Em relação às características das disciplinas sobre PICs ofertadas pelas instituições, apenas 31 (18\%) apresentaram na grade curricular do curso disciplinas relacionadas a PICs, destas disciplinas ofertadas, 17 (9,9\%) eram obrigatórias, $66(38,4 \%)$ presenciais e com a média da carga horária 403,6 horas (DP = 411,8) (Tabela 2).

Entre as disciplinas ofertadas, 16 $(51,6 \%)$ eram de cursos de pós-graduação lato sensu entre as quais 11 sobre acupuntura, duas sobre fitoterapia clínica, uma sobre aromaterapia, uma fitoterapia chinesa, uma sobre naturopatia e uma sobre práticas integrativas e complementares em saúde. Nos cursos de graduação, foram identificadas 15 (48,4\%) disciplinas, dentre as quais nove sobre práticas integrativas em geral, duas sobre Mindfulness para Qualidade de Vida e Redução de Estresse, uma sobre acupuntura e uma sobre cuidados paliativos e terapias alternativas. Entre os cursos de graduação que ofereciam disciplinas sobre PICs 14 (93,3\%) eram bacharel em Enfermagem e um $(6,7 \%)$ bacharel e licenciatura em Enfermagem. 


\section{DISCUSSÃO}

Com os resultados deste estudo foi possível identificar que apesar da implementação da PNPIC desde 2006 no SUS, a temática das PICs ainda é incipiente em disciplinas dos cursos de graduação em Enfermagem do Estado de São Paulo(2). Uma vez que os resultados deste estudo mostram um déficit na oferta de disciplinas sobre PICs nos cursos de graduação em Enfermagem no Estado de São Paulo, como também nos cursos de pós-graduação lato sensu, pois das 172 instituições de ensino identificadas apenas 31 (18\%) oferecem disciplinas sobre a temática.

No que tange aos cursos de pós-graduação lato sensu na área da Enfermagem, o número de instituições que disponibilizam disciplinas com foco em PICs pouco difere dos resultados apresentados nos cursos de graduação, visto que das 31 instituições identificadas que oferecem disciplinas sobre PICs 16 (51,6\%) eram de cursos de pós-graduação lato sensu.

Um estudo realizado em 2014 avaliou a oferta de disciplinas sobre PICs em universidades públicas no Brasil dos cursos de Medicina, Enfermagem e Fisioterapia. As instituições de Enfermagem foram as que mais ofereceram disciplinas, sendo que entre as 87 instituições públicas de graduação em Enfermagem no Brasil 23 (26,4\%) apresentaram disciplinas sobre PICs, dentre as quais oito (35\%) estão localizadas no nordeste do país, em seis instituições (26,1\%) as disciplinas tinham caráter obrigatório e média de carga horária de 44 horas $^{(11)}$.

Um estudo realizado no Estado do Rio de Janeiro avaliou 15 subáreas da Saúde, dentre elas 39 (85\%) graduações e sete (15\%) pós-graduações, três cursos de especialização lato sensu, cinco projetos de extensão e duas ligas acadêmicas com um total de 56 instituições que realizaram a oferta de disciplinas sobre PICs. Entre as áreas avaliadas, a Medicina apresentou maior frequência de disciplinas sobre PICs com $31 \%$, em seguida o curso de Farmácia com $22 \%$, a Enfermagem com $14 \%$, a Terapia Ocupacional com 7\%, Educação
Física, Psicologia e Saúde Coletiva com 5\% cada e a Medicina Veterinária com $4 \%$. Os cursos de Fonoaudiologia, Odontologia, Biomedicina e Ciências Biológicas apresentaram juntos apresentam 7\% entre os cursos das instituições públicas do Estado do Rio de Janeiro que oferecem disciplinas sobre PICs. Os resultados do presente estudo demonstram que comparativamente aos resultados do estudo realizado no Estado do Rio de Janeiro, o Estado de São Paulo apresenta um número maior de cursos de graduação em Enfermagem que oferecem disciplinas sobre PICs com 15 (48,4\%) instituições. No entanto, é relevante destacar que o número de instituições de ensino no Estado de São Paulo é maior e que foram incluídas também as instituições privadas de ensino ${ }^{(8)}$.

$\mathrm{Na}$ Espanha, foi identificado que no curso de Enfermagem, na modalidade licenciatura, entre 94 escolas de Enfermagem, centros e faculdades analisadas, em $20(21,28 \%)$ apresentavam disciplinas sobre as PICs. No entanto, dez das 20 disciplinas foram aprovadas, porém tinham a implementação pendente para futuros anos letivos. Em relação ao conteúdo das disciplinas sobre PICs, abordavam reflexologia podal, florais de Bach, relaxamento e hidroterapia, acupuntura, massagem, toque terapêutico, terapia do riso, aromaterapia, fitoterapia, Reiki, Yoga e musicoterapia ${ }^{(12)}$. Comparativamente, no presente estudo, foi identificado nos cursos de graduação disciplinas sobre acupuntura, cuidados paliativos e terapias alternativas e Mindfulness para Qualidade de Vida e Redução de Estresse.

Uma revisão integrativa sobre as estratégias de ensino das terapias complementares nos cursos de graduação em Enfermagem identificou que as propostas para inserção desta temática na área acadêmica ocorrem em geral por meio de palestras, cursos teóricos e grupo de discussão, disciplina optativa, como também, inserção de disciplina no currículo e incentivo à pesquisa. Foi pontuado pelos autores que para que viabilize de fato a inclusão sobre PICs no Projeto Po- lítico Pedagógico do curso de graduação em Enfermagem ainda são necessários o desenvolvimento de mais estudos que comprovem a eficácia dessas terapias ${ }^{(13)}$.

Em geral, a formação sobre as diferentes PICs acontece ao longo da carreira do enfermeiro, em cursos de diferentes formatos, mas com caráter de educação continuada e especialização, não ocorrendo, portanto, um momento específico que possa indicar uma possível associação com os questionamentos da formação profissional. Nas cinco escolas de Enfermagem avaliadas em estudo, foi identificado que o processo de introdução das PICs seguiu o seguinte fluxo: exposição às práticas, o uso pessoal e a possibilidade do ensino e pesquisa. É relevante considerar esses aspectos, principalmente porque estimulam os estudantes e futuros profissionais da enfermagem a aprenderem sobre as PICs com menor ceticismo e preconceito ${ }^{(14)}$.

Por não ser uma prática muito difundida no âmbito hospitalar, a maioria das instituições de ensino não inclui as PICs nas grades curriculares, mesmo que essas práticas tenham sido regulamentadas pelo SUS, tendo como foco principal a prevenção dos agravos de doenças crônicas e um dos meios estratégicos da atenção básica para prevenção dessas doenças. A falta de investimento nos cursos sobre as PICs pode causar um déficit no número de profissionais capacitados para realizar tais práticas. O Ministério da Saúde manifesta essa dificuldade em seu manual de implantação do serviço PICs no SUS, ressaltando que uma de suas estratégias é desenvolver a oferta de cursos para os gestores e profissionais da saúde para meIhorar o suporte nesta área ${ }^{(15)}$. Um estudo realizado no Estado da Paraíba avaliou o conhecimento dos enfermeiros que atuam na atenção básica sobre a PNPIC identificando que a maioria desconhece este plano devido à lacuna no processo de formação e na falta de educação permanente por meio de cursos, o que reflete na falta de embasamento científico sobre as PICs ${ }^{(16)}$.

Ainda que a formação dos enfermeiros no Brasil, de acordo com as Diretrizes Curriculares Nacionais, seja baseada em 
uma formação generalista, seria relevante incluir essa temática no ensino da enfermagem, pois a escassez de profissionais capacitados é apontada como um dos desafios enfrentados pelo Ministério da Saúde na implantação efetiva das PICs na rede de atenção básica do $\operatorname{SUS}{ }^{(15,17)}$.

Sendo assim, foi identificado no presente estudo, assim como em outros estudos $^{(8,11)}$ que a maioria dos cursos de graduação e pós-graduação não oferece disciplinas que proporcionem o conhecimento básico sobre PICs para a atuação do enfermeiro nesta área. Apesar da enfermagem em práticas integrativas e complementares ser um dos cursos de pós-graduação lato sensu reconhecido e regulamentado pelo COFEN abrangendo as práticas de: fitoterapia, homeopatia, ortomolecular, terapia floral, reflexologia podal, reiki, yoga, toque terapêutico, musicoterapia, cromoterapia, hipnose e acupuntura(18).

Este estudo apresenta limitações, pois avaliou base de dados on-line e, muitas vezes, os dados podem não estar atualizados ou não estarem disponíveis, principalmente das instituições privadas de ensino.

\section{CONCLUSÃO}

Por meio deste estudo foi possível identificar que embora as PICs estejam preconizadas nas políticas do SUS e pelo COFEN, ainda há uma escassez de instituições públicas e privadas do Estado de São Paulo que oferecem disciplinas nos cursos de graduação sobre essas práticas, além de cursos de pós-graduação lato sensu. Embora as diretrizes curriculares dos cursos de graduação em Enfermagem tenham como objetivo formar o enfermeiro com formação generalista, ressalta-se a relevância em incluir disciplinas sobre PICs ainda que sejam como disciplinas optativas com a finalidade de oferecer a oportunidade aos estudantes de conhecerem sobre essa área de atuação.

\section{Referências}

1. Brasil. Lei n. ${ }^{\circ} 8.080$, de 19 de setembro de 1990 . Dispõe sobre as condições para a promoção, proteção e recuperação da saúde, a organização e o funcionamento dos serviços correspondentes e dá outras providências [Internet]. 1990 [acesso em 2019 abr 20]. Disponivel em: http://www.planalto.gov.br/ ccivil_03/leis//8080.htm.

2. Ministério da Saúde, Secretaria de Atenção à Saúde, Departamento de Atenção Básica (BR). Política Nacional de Práticas Integrativas e Complementares no SUS - PNPIC-SUS [Internet]. 2006 [acesso em 2019 abr 20]. Disponível em: http://bvsms.saude.gov.br/bvs/publicacoes/pnpic.pdf.

3. Ministério da Saúde (BR). Portaria n. ${ }^{\circ} 849$, de 27 de março de 2017. Diário Oficial da República Federativa do Brasil. Política Nacional de Práticas Integrativas e Complementares (PNPIC) [Internet]. 2017 [acesso em 2019 abr 29]. Disponível em: http://bvsms.saude.gov.br/bvs/saudelegis/gm/2017/ prt0849_28_03_2017.html.

4. Ministério da Saúde (BR). Portaria n. ${ }^{\circ}$ 702, de 21 de março de 2018. Para incluir novas práticas na Política Nacional de Práticas Integrativas e Complementares (PNPIC) [Internet]. 2018 [acesso em 2019 mai 05]. Disponível em: http://138.68.60.75/images/portarias/marco2018/dia22/portaria702.pdf.

5. Conselho Federal de Enfermagem (BR). Resolução COFEN n. ${ }^{\circ} 0500 / 2015$. Reconhecimento de Terapias Alternativas como especialidade e/ou qualificação do profissional de Enfermagem [Internet]. 2015 [acesso em 2019 mai 12]. Disponível em: http://www.cofen.gov.br/resolucao-cofen-no-05002015_36848.html. 6. Conselho Federal de Enfermagem (BR). Resolução COFEN n. ${ }^{\circ}$ 581/ 2018. Procedimentos para Registro de Títulos de Pós-Graduação Lato e Stricto Sensu concedido a Enfermeiros e aprova a lista das especialidades [Internet]. 2018 [acesso em 2019 mai 12]. Disponível: http://www.cofen.gov.br/ wp-content/uploads/2018/07/RESOLU\%C3\%87\%C3\%830-COFEN-N\%C2\%BA-0581-2018.pdf

7. Salles LF, Homo RFB, Silva MJP. Práticas Integrativas e Complementares: situação do seu ensino na graduação de enfermagem no Brasil. Rev. Saúde [Internet]. 2014 [acesso em 2019 jun 16]; 8 (3-4): 37-44. Disponível em: http:// revistas.ung.br/index.php/saude/article/view/2005/1579.

8. Nascimento DCM, Romano FV, Chazan SCA, Quaresma HC. Formação em práticas integrativas e complementares em saúde: desafios para as universidades públicas. Trab. educ. saúde [Internet]. 2018 [acesso em 2019 jun 16]; 16(2): 751-772. Disponível em: http://dx.doi.org/10.1590/1981-7746-sol00130.

9. Ministério da Educação (BR). Cadastro Nacional de Cursos e Instituições de Educação Superior Cadastro e-MEC. [Internet]. [acesso em 2019 jul 20]. Disponível em: http://emec.mec.gov.br/.
10. Ministério da Saúde, Conselho Nacional de Saúde (BR). Ética na Pesquisa. Resolução 510, de 07 de abril de 2016 [Internet]. 2016 [acesso em 2019 jun 20]. Disponivel em: http://conselho.saude.gov.br/resolucoes/2016/Reso510.pdf. 11. Salles FL, Homo BFR, Silva DPJM, Situação do ensino das práticas integrativas e complementares nos cursos de graduação em enfermagem, fisioterapia e medicina. Cogitare enferm [Internet]. 2014 [acesso em 2019 ago 16]; 19 (4): 742-746. Disponível em: http://www.revenf.bvs.br/scielo.php?script=sci_arttext\&pid=S1414-85362014000400013.

12. Fernández-Cervilla $A B$, Piris-Dorado Al, Cabrer-Vives ME, Barquero-González A. Estado atual do ensino de Terapias Complementares na formação superior de Enfermagem na Espanha. Rev. latinoam. enferm. [Internet]. 2013 [acesso em 2019 ago 20]; 21 (3): 679-686. Disponível em: http://dx.doi. org/10.1590/S0104-11692013000300005.

13. Silva N, lunes D, Resck Z, Soares M., Souza Junior D, Vieira, N. Estratégias de ensino das terapias alternativas e complementares na graduação em Enfermagem: revisão integrativa. Rev. eletrônica enferm. [Internet]. 2013 [acesso em 2019 set 02]; 15(4): 1061-1067. Disponível em: https://doi.org/10.5216/ ree.v15i4.20568.

14. Barros NF, Tovey P. 0 ensino das terapias alternativas e complementares em escolas de enfermagem. Rev. gaúch. enferm. [Internet]. 2007 [acesso em 2019 set 20]; 28(2):207-214. Disponivel em: https://seer.ufrgs.br/RevistaGauchadeEnfermagem/article/view/3165/1736.

15. Ministério da Saúde, Secretaria de Atenção à Saúde, Departamento de Atenção Básica (BR). Manual de implantação de serviços de práticas integrativas e complementares no SUS [Internet]. 2018. [acesso em 2019 out 20]. Disponivel em: http://189.28.128.100/dab/docs/portaldab/publicacoes/manual_implantacao_servicos_pics.pdf.

16. Soares DP, Coelho AM, Silva LEAS, et al. Política Nacional de práticas integrativas e complementares em saúde: Discurso dos enfermeiros da Atenção Básica. Rev. enferm. Centr-Oeste Min. [Internet]. 2019 [acesso em 2019 out 29];9:e3265. Disponivel em: http://dx.doi.org/10.19175/ recom.v9i0.3265.

17. Ministério da Educação (BR). Diretrizes curriculares nacionais do curso de graduação em Enfermagem. [Internet]. 2001. [acesso em 2019 nov 05]. Disponível em: http://portal.mec.gov.br/cne/arquivos/pdf/CES03.pdf.

18. Conselho Federal de Enfermagem (BR). Resolução COFEN n. ${ }^{\circ}$ 570/2018. Resolução COFEN No 570/2018 - Revogada pela resolução COFEN n. ${ }^{\circ}$ 577/2018 [Internet]. 2018. [acesso em 2019 nov 30]. Disponível em: http:// www.cofen.gov.br/resolucao-cofen-no-0570-2018_61172.html. 